2004

\title{
Vacancy Defects In O-Doped Gan Grown By Molecular-Beam Epitaxy: The Role Of Growth Polarity And Stoichiometry
}

M. Rummukainen

J. Oila

A. Laakso

K. Saarinen

A.J.Ptak

See next page for additional authors

Follow this and additional works at: https://researchrepository.wvu.edu/faculty_publications

\section{Digital Commons Citation}

Rummukainen, M.; Oila, J.; Laakso, A.; Saarinen, K.; Ptak, A. J.; and Myers, T. H., "Vacancy Defects In O-Doped Gan Grown By Molecular-Beam Epitaxy: The Role Of Growth Polarity And Stoichiometry" (2004). Faculty Scholarship. 724.

https://researchrepository.wvu.edu/faculty_publications/724 
Authors

M. Rummukainen, J. Oila, A. Laakso, K. Saarinen, A. J. Ptak, and T. H. Myers 


\title{
Vacancy defects in 0-doped GaN grown by molecular-beam epitaxy: The role of growth polarity and stoichiometry
}

\author{
M. Rummukainen, ${ }^{\text {a) }}$ J. Oila, A. Laakso, and K. Saarinen \\ Laboratory of Physics, Helsinki University of Technology, P.O. Box 1100 FIN-02015 HUT, Finland
}

A. J. Ptak and T. H. Myers

Department of Physics, West Virginia University, Morgantown, West Virginia 26506

(Received 29 December 2003; accepted 19 April 2004; published online 25 May 2004)

\begin{abstract}
Positron annihilation spectroscopy is used to study vacancy defects in GaN grown by molecular-beam epitaxy due to different polar directions and varying stoichiometry conditions during oxygen doping. We show that Ga-polar material is free of compensating Ga vacancies up to $[\mathrm{O}]=10^{18} \mathrm{~cm}^{-3}$ in $\mathrm{Ga}$ stable growth, but high concentrations of $V_{\mathrm{Ga}}$ are formed in N-stable conditions. We also show that vacancy clusters are formed in N-polar material grown in Ga stable conditions, which may be related to the higher reactivity of the $\mathrm{N}$-polar surface. These clusters have no apparent influence on the electrical properties of the material. We thus infer that their charge state is neutral. (C) 2004 American Institute of Physics. [DOI: 10.1063/1.1762984]
\end{abstract}

Properties of gallium nitride depend strongly on the crystal orientation and the polarity of the growth direction. Growth in the Ga-polar (0001) direction generally leads to smooth surface morphology and high quality material, while $\mathrm{N}$-polar $(000 \overline{1})$ growth is much more difficult, though it may be desirable since it is more easily etched. ${ }^{1,2}$ The stoichiometry of $\mathrm{Ga}$ and $\mathrm{N}$ overpressures during the growth is also important. ${ }^{1}$ Generally molecular-beam epitaxy (MBE) of $\mathrm{GaN}$ takes place in Ga stable conditions, where the diffusivity of $\mathrm{Ga}$ adatoms is high at the surface. ${ }^{1,3}$ Approaching $\mathrm{N}$ stable growth, however, the surface mobility of Ga adatoms is reduced due to $\mathrm{Ga}-\mathrm{N}$ bonds ${ }^{1,3}$ and surface reactivity is increased enhancing oxygen incorporation by orders of magnitude. .,5 $^{4}$

Experimental and theoretical studies demonstrate the influence of growth polarity and stoichiometry on the impurity incorporation. $^{4-6}$ The epitaxial N-polar GaN contains typically an order of magnitude more oxygen than Ga-polar layers, and approaching N-stable conditions the oxygen incorporation is strongly enhanced for both polarities. ${ }^{5}$ In $\mathrm{Ga}$ polar samples, controlled oxygen doping without significant electrical compensation can be achieved up to [O] $\cong 10^{18} \mathrm{~cm}^{-3}$, but at higher doping levels electrically compensating defects are formed. ${ }^{5}$ The effect of growth polarity and stoichiometry on the formation of point defects is much less studied and thus less well understood than on the impurity incorporation.

In this work we apply positron annihilation spectroscopy to study the formation of vacancy defects in GaN grown in different polar orientations and under different stoichiometry conditions. We confirm that Ga-polar material is free of compensating $\mathrm{Ga}$ vacancies at moderate $\mathrm{O}$-doping levels for $\mathrm{Ga}$ stable growth, but a high concentration of $V_{\mathrm{Ga}}$ is formed in $\mathrm{N}$-stable conditions. We also show that vacancy clusters are formed in N-polar GaN grown in Ga stable stoichiometry, but these clusters are likely to be electrically inactive.

The O-doped GaN layers (Table I) were grown at West

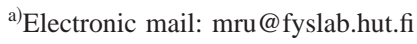

Virginia University (WVU) by rf plasma-assisted MBE, as described earlier. ${ }^{5,7}$ The N-polarity GaN was grown by nucleating $\mathrm{GaN}$ buffer layers directly on sapphire in highly Ga-rich conditions. The Ga-polarity $\mathrm{GaN}$ was prepared by epitaxy on (0001) GaN templates grown by metalorganic chemical vapor deposition (MOCVD) on A-plane sapphire at the Naval Research Laboratory. The stoichiometry was varied by changing the beam-equivalent pressure (BEP) of Ga, as shown in Table I.

The positron experiments were performed with a monoenergetic beam by measuring the Doppler broadening of the $511 \mathrm{keV}$ annihilation radiation. ${ }^{8}$ The Doppler spectra were analyzed with the conventional $S$ and $W$ parameters, describing positron annihilations with valence and core electrons, respectively. When thermalized positrons are trapped at vacancy defects, the reduced electron density leads to an increase in $S$ and decrease in $W$ parameters. ${ }^{8}$

In the MBE layers, the $S$ parameter is high at low positron implantation energies, $E<5 \mathrm{keV}$, due to positron annihilations on the sample surface (Fig. 1). When the energy is increased the annihilations take place inside the GaN layer,

TABLE I. The polarity, thickness, Ga-beam equivalent pressure, O-partial pressure, and dopant concentration in the studied MBE-grown layers.

\begin{tabular}{lccccc}
\hline \hline $\begin{array}{c}\text { Polarity and } \\
\text { thickness }(\mu \mathrm{m})\end{array}$ & $\begin{array}{c}\mathrm{BEP}(\mathrm{Ga}) \\
\left(10^{-6} \text { Torr }\right)\end{array}$ & $\begin{array}{c}p_{\mathrm{O}_{2}} \\
(\text { Torr })\end{array}$ & $\begin{array}{c}{[\mathrm{O}]} \\
\left(\mathrm{cm}^{-3}\right)\end{array}$ & $\begin{array}{c}n \\
\left(\mathrm{~cm}^{-3}\right)\end{array}$ \\
\hline$\# 1 \mathrm{~N}$ & 1 & 1.6 & $4.0 \times 10^{-12}$ & & $1.0 \times 10^{15}$ \\
$\# 2 \mathrm{~N}$ & 1 & 0.96 & $5.0 \times 10^{-10}$ & & $1.7 \times 10^{18}$ \\
$\# 3 \mathrm{~N}$ & 1 & 1.4 & $4.0 \times 10^{-11}$ & $3.0 \times 10^{16}$ & $6.0 \times 10^{16}$ \\
$\# 4 \mathrm{~N}$ & 1 & 1.6 & $4.0 \times 10^{-11}$ & $3.0 \times 10^{16}$ & $6.6 \times 10^{16}$ \\
$\# 5 \mathrm{~N}$ & 1 & 0.5 & $1.0 \times 10^{-7}$ & & $6.8 \times 10^{19}$ \\
$\# 6 \mathrm{Ga}$ & 1.8 & 0.49 & $1.5 \times 10^{-7}$ & $2.5 \times 10^{22}$ & $3.0 \times 10^{20}$ \\
$\# 7 \mathrm{Ga}$ & 1.8 & 0.46 & $5.5 \times 10^{-8}$ & & $9.0 \times 10^{19}$ \\
$\# 8 \mathrm{Ga}$ & 1 & 0.48 & $5.0 \times 10^{-8}$ & $2.6 \times 10^{21}$ & $4.0 \times 10^{17}$ \\
$\# 9 \mathrm{Ga}$ & 1.36 & 0.49 & $2.0 \times 10^{-7}$ & $1.5 \times 10^{22}$ & $7.4 \times 10^{19}$ \\
$\# 10 \mathrm{Ga}$ & 0.62 & 0.95 & $1.1 \times 10^{-7}$ & $1.5 \times 10^{18}$ & $1.4 \times 10^{18}$ \\
$\# 11 \mathrm{Ga}$ & 1.9 & 1.2 & $4.0 \times 10^{-12}$ & $<1 \times 10^{16}$ & $3.8 \times 10^{17}$ \\
$\# 12 \mathrm{Ga}$ & 0.9 & 0.93 & $1.0 \times 10^{-9}$ & $5.0 \times 10^{16}$ & $2.8 \times 10^{16}$ \\
$\# 13 \mathrm{Ga}$ & 1 & 1.6 & $3.0 \times 10^{-11}$ & & $3.0 \times 10^{17}$ \\
$\# 14 \mathrm{Ga}$ & 1 & 0.73 & $2.0 \times 10^{-10}$ & $1.0 \times 10^{16}$ & $1.2 \times 10^{16}$ \\
$\# 15 \mathrm{Ga}$ & 1 & 1 & $2.0 \times 10^{-8}$ & $3.0 \times 10^{17}$ & $3.0 \times 10^{17}$ \\
\hline \hline
\end{tabular}




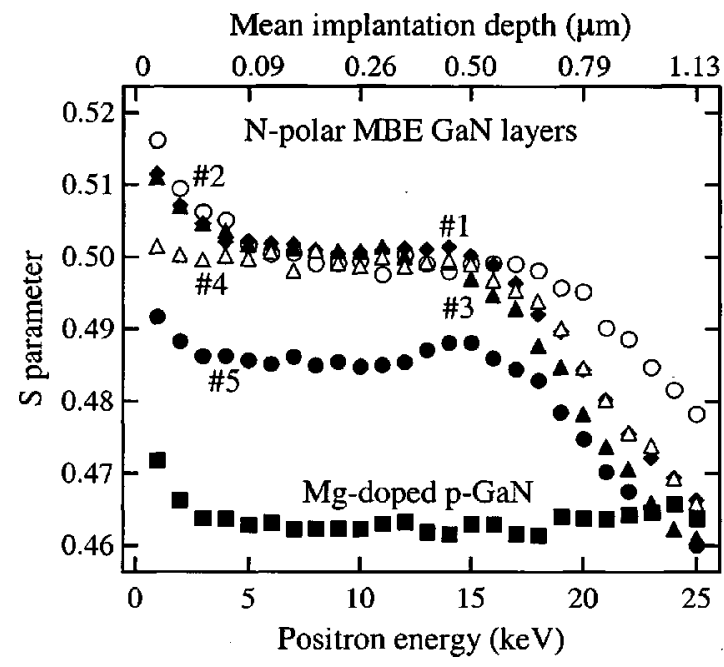

FIG. 1. The $S$ parameter as a function of positron incident energy in the studied N-polar GaN layers and in the $p$-type GaN:Mg layer. The mean penetration depth is indicated by the top axis.

seen as a constant level in the $S$ parameter. At $E>17 \mathrm{keV}$ positrons reach the underlying $\mathrm{Al}_{2} \mathrm{O}_{3}$ substrate layer and the $S$ parameter decreases $\left(S_{\mathrm{Al}_{2} \mathrm{O}_{3}} \approx 0.43\right)$. Annihilation parameters in the $p$-type $\mathrm{Mg}$-doped $\mathrm{GaN}$ reference layer correspond to vacancy-free GaN lattice. ${ }^{9}$ In the N-polar samples 1-4 the $S$ parameters characterizing the MBE layer are approximately the same while in the N-polar sample 5 the $S$ parameter is clearly lower. However, the $S$ parameters in all the N-polar layers are clearly higher than in a vacancy-free GaN lattice, $S_{b} \approx 0.463$, indicating positron trapping to vacancy type defects.

The $S$ parameter curves in the Ga-polar samples (Fig. 2) show two kinds of behavior. A plateau characterizing the MBE layer is clearly distinguishable only in samples 6-9. The high layer specific $S$ parameter, $S \approx 0.485$, indicates trapping to vacancy type defects. In the other Ga-polar samples, Nos. $10-15$, the $S$ parameter continues to decrease without a clear plateau from the surface value toward $S \approx 0.465$, which was recorded in the MOCVD GaN below the MBE layer.

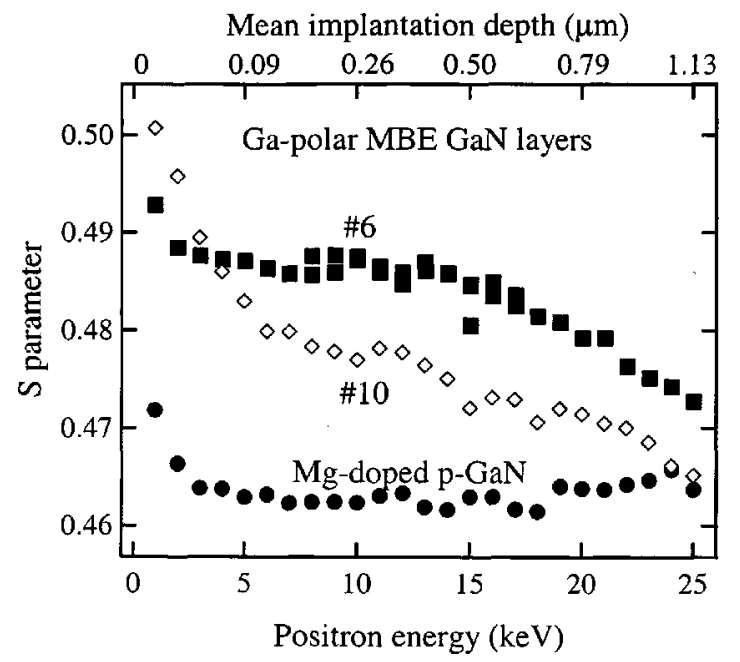

FIG. 2. The $S$ parameter as a function of positron incident energy in two Ga-polar GaN layers. The $S$ parameter characterizing the MBE layer is distinguishable in sample 6, while in sample 10 annihilations in the MBE layer are mixed with annihilations on the surface and the MOCVD-GaN substrate.

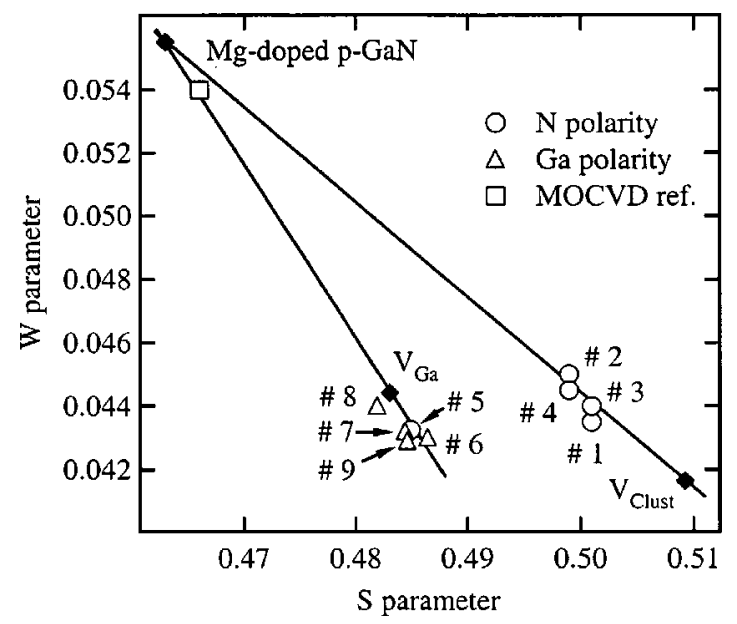

FIG. 3. The $S-W$ plot of $(S, W)$ values characterizing the MBE layers. The $(S, W)$ values corresponding to vacancy free $\mathrm{GaN}\left(S_{b}, W_{b}\right)$, Ga vacancy $\left(S_{V_{\mathrm{Ga}}}, W_{V_{\mathrm{Ga}}}\right)$, and vacancy cluster $\left(S_{\text {clust }}, W_{\text {clust }}\right)$ are also shown.

The annihilation parameters specific to the MBE layer cannot thus be estimated in these samples.

The number of different vacancy type defects can be studied by plotting the $S$ parameters as a function of $W$ parameters (Fig. 3). ${ }^{8}$ The data points from N-polar samples 1-4 are clearly separate from the Ga-polar samples 6-9 and the N-polar sample 5, indicating the vacancy defects in these two sample groups are different. Positron studies have previously identified two types of vacancy defects: Ga vacancies, ${ }^{10}$ with characteristic parameters $S_{V_{\mathrm{Ga}}} / S_{b} \approx 1.046$ and $W_{V_{\mathrm{Ga}}} / W_{b} \approx 0.80$, and vacancy clusters with an open volume of a divacancy or bigger, ${ }^{11,12}$ characterized by $S_{\text {clust }} / S_{b} \approx 1.10$ and $W_{\text {clust }} / W_{b} \approx 0.75$. In Fig. 3 the $(S, W)$ values from the Ga-polar samples 6-9 and the N-polar sample 5 fall very close to the point characteristic of the $\mathrm{Ga}$ vacancy, suggesting a high $V_{\mathrm{Ga}}$ concentration. On the other hand, the slope of the line between the $(S, W)$ points of the $\mathrm{N}$-polar samples 1-4 and the vacancy-free lattice $\left(S_{b}, W_{b}\right)$ coincides very well with that characteristic to the vacancy cluster. The positron trapping at the vacancy clusters is also suggested by the very high $S$ parameter, $S / S_{b} \approx 1.08$.

Interestingly, the $\mathrm{Ga}$ vacancies are detected in very high concentrations both in Ga-polar and the N-polar samples where the gallium BEP is low, $\sim 0.5 \times 10^{-6}$ Torr. This means the growth approaches $\mathrm{N}$-stable conditions. ${ }^{5}$ Note that this also significantly enhances oxygen incorporation. The $S$ and $W$ values do not significantly differ from $S_{V_{\mathrm{Ga}}}$ and $W_{V_{\mathrm{Ga}}}$, i.e., positron trapping is in saturation. Therefore, it is only possible to give a lower limit estimate, $\left[V_{\mathrm{Ga}}\right] \geq 5 \times 10^{18} \mathrm{~cm}^{-3}$. 8 It is likely that these vacancies are charged, forming the compensating defects that limit the carrier concentration obtainable from the oxygen. The charged nature is reflected in the extremely low mobility, $\leqslant 20 \mathrm{~cm}^{2} / \mathrm{V} \mathrm{s}$ at $300 \mathrm{~K}$, in the samples 5-9.

The presence of the high $V_{\mathrm{Ga}}$ concentration in the samples with lower gallium BEP is very reasonable. It agrees with the previous results, where the $V_{\mathrm{Ga}}$ concentration was found to increase in undoped ( $n$-type) MOCVD GaN layers with the increasing V/III-molar ratio. ${ }^{13}$ According to Ref. 5, the incorporation of oxygen is significantly increased both in Ga-polar and N-polar growth direction when the growth is 
$\mathrm{N}$-stable. The observed high $V_{\mathrm{Ga}}$ concentration in the $\mathrm{N}$-stable samples thus agrees with the theoretical calculations, predicting the formation of compensating $V_{\mathrm{Ga}}$ should be enhanced in the strongly $n$-type material. ${ }^{14,15}$

The positron annihilation experiments in irradiated GaN have shown that the isolated $V_{\mathrm{Ga}}$ are mobile and recover by migration already at $300{ }^{\circ} \mathrm{C} .{ }^{16}$ Thus the observed vacancies are most probably $V_{\mathrm{Ga}}$-donor-impurity pairs $\left(V_{\mathrm{Ga}}-\mathrm{O}_{\mathrm{N}}\right.$ in this case), which can survive the growth temperature. The presence of the Ga vacancies only in the samples with the high oxygen content ( $\mathrm{N}$-stable growth) manifests the essential role of oxygen both in decreasing the formation energy of $\mathrm{Ga}$ vacancies and enhancing their stability. ${ }^{9}$

In Ga-polar layers grown in Ga-stable conditions (samples 10-15) the annihilation parameters (Fig. 2) show a smooth transition from the surface values toward those of GaN lattice, indicating a positron diffusion length of about $100 \mathrm{~nm}$. The long positron diffusion length and the absence of apparent signs of vacancies indicate that at the most $\left[V_{\mathrm{Ga}}\right]$ is in the $10^{16} \mathrm{~cm}^{-3}$ range. This is in good agreement with the electrical properties which show very little compensation. ${ }^{5}$ Controlled oxygen doping can thus be done up to the level of $10^{18} \mathrm{~cm}^{-3}$ without significant formation of compensating $\mathrm{Ga}$ vacancies.

The observation of the vacancy clusters in the N-polar layers grown in Ga-stable conditions is very interesting. According to the depth scan of Fig. 1, the vacancy clusters are found throughout the MBE layer. The concentration of the clusters can be estimated to be in the $10^{17} \mathrm{~cm}^{-3}$ range. The positron data do not give evidence of the charge state of the vacancy cluster. However, two results suggest the observed vacancies may be electrically neutral. First, there is a close correspondence between the oxygen as measured by secondary ion mass spectroscopy (SIMS) and the carrier concentration measured by Hall experiments for these and other $\mathrm{N}$-polar samples, ${ }^{5}$ particularly below the $10^{18} \mathrm{~cm}^{-3}$ range. Second there is little difference between the measured Hall mobilities for samples 3 and 4 with the high vacancy cluster content, and samples 12 and 14 with no detectable vacancy cluster content. Room temperature mobilities for these samples were between 350 and $400 \mathrm{~cm}^{2} / \mathrm{V} \mathrm{s}$, and all four mobilities peaking at $\sim 800 \mathrm{~cm}^{2} / \mathrm{V} \mathrm{s}$ at lower temperature. The dislocation scattering likely plays a significant role in limiting the mobility. ${ }^{17}$

Positron trapping at similar vacancy clusters has been observed earlier in MBE GaN layers (unknown growth polarity) grown on $\mathrm{Si}(111)$ substrate $^{12}$ and in Si-doped MBE layers grown on HVPE-GaN substrate ${ }^{11}$ (Ga-polar, but inversion boundaries were seen by electron microscopy). Also Uedono et al. have reported positron trapping at larger vacancy clusters in N-polar MBE-grown GaN layer. ${ }^{18}$ It thus seems that the formation of clusters is related to the N-polar growth direction. Though obtaining high quality growth on the N-polar surface is harder, the layers of this work had a comparable surface morphology and electrical quality to Gapolar growth. The higher reactivity of the $(000 \overline{1})$ surface enhances the incorporation of oxygen impurities, ${ }^{5}$ but also enables the formation of vacancy clusters. This may be one of the limiting factors for N-polar MBE-growth of GaN.

The formation of vacancy cluster requires empty $\mathrm{Ga}$ and
$\mathrm{N}$ lattice sites. In addition to $V_{\mathrm{Ga}}$, which always has a low formation energy in $n$-GaN, ${ }^{14,15}$ perhaps $\mathrm{N}$ vacancies could form under $\mathrm{Ga}$ stable condition (i.e., favorable stoichiometry) especially in $\mathrm{N}$ polar growth since this has a larger number of $\mathrm{N}$ sites that can be left empty. $V_{\mathrm{Ga}}$ and $V_{\mathrm{N}}$ can form cluster by mobility of at least one of the species at the growth temperature, especially since $V_{\mathrm{Ga}}$ and $V_{\mathrm{N}}$ are attracted by their opposite charges.

In conclusion, we observe positron trapping at two kinds of vacancy type defects in MBE-grown O-doped GaN; a Ga vacancy, or more likely the $V_{\mathrm{Ga}}-\mathrm{O}_{\mathrm{N}}$ complex, and a vacancy cluster. When the growth is done in Ga-stable conditions, leading to a low or moderate O-doping, vacancy clusters are formed in N-polar GaN, while Ga-polar layers show no significant vacancy concentrations. The absence of vacancies in the low-doped Ga-polar samples is consistent with electrical data indicating negligible compensation. However, when the growth is $\mathrm{N}$-stable, leading to a high oxygen doping, the $\mathrm{Ga}$ vacancy becomes the dominating defect in the both growth directions, suggesting that oxygen impurities have a crucial role in the formation of $\mathrm{Ga}$ vacancies.

The authors would like to thank A. E. Wickenden, D. D. Koleske, and R. L. Henry at the Laboratory of Advanced Material Synthesis, Naval Research Laboratory for providing the MOCVD GaN templates. Work at WVU was supported by ONR Grant Nos. N00014-02-1-0974 and N00014-010571, monitored by Colin Wood.

${ }^{1}$ E. J. Tarsa, B. Heying, X. H. Wu, S. P. DenBaars, and J. S. Speck, J. Appl. Phys. 82, 5472 (1997).

${ }^{2}$ M. Seelmann-Eggebert, J. L. Weyher, H. Obloh, H. Zimmermann, A. Rar, and S. Porowski, Appl. Phys. Lett. 71, 2635 (1997).

${ }^{3}$ T. Zywietz, J. Neugebauer, and M. Scheffler, Appl. Phys. Lett. 73, 487 (1998).

${ }^{4}$ T. Zywietz, J. Neugebauer, and M. Scheffler, Appl. Phys. Lett. 74, 1695 (1999).

${ }^{5}$ A. J. Ptak, L. J. Holbert, L. Ting, C. H. Swartz, M. Moldovan, N. C. Giles, T. H. Myers, P. Van Lierde, C. Tian, R. A. Hockett, S. Mitha, A. E. Wickenden, D. D. Koleske, and R. L. Henry, Appl. Phys. Lett. 79, 2740 (2001).

${ }^{6}$ A. L. Rosa, J. Neugebauer, J. E. Northrup, C. D. Lee, and R. M. Feenstra, Appl. Phys. Lett. 80, 2008 (2002).

${ }^{7}$ T. H. Myers, L. S. Hirsch, L. T. Romano, and M. R. Richards-Babb, J. Vac. Sci. Technol. B 16, 2261 (1998).

${ }^{8}$ K. Saarinen, P. Hautojärvi, and C. Corbel, in Identification of Defects in Semiconductors, edited by M. Stavola (Academic, New York, 1998).

${ }^{9}$ J. Oila, V. Ranki, J. Kivioja, K. Saarinen, P. Hautojärvi, J. Likonen, J. M. Baranowski, K. Pakula, T. Suski, M. Leszczynski, and I. Grzegory, Phys. Rev. B 63, 045205 (2001).

${ }^{10}$ K. Saarinen, T. Laine, S. Kuisma, J. Nissilä, P. Hautojärvi, L. Dobrzyniski, J. M. Baranowski, K. Pakula, R. Stepniewski, M. Wojdak, A. Wysmolek, T. Suski, M. Leszczynski, I. Grzegory, and S. Porowski, Phys. Rev. Lett. 79, 3030 (1997)

${ }^{11}$ P. Laukkanen, S. Lehkonen, P. Uusimaa, M. Pessa, J. Oila, S. Hautakangas, K. Saarinen, J. Likonen, and J. Keränen, J. Appl. Phys. 92, 786 (2002).

${ }^{12}$ E. Calleja, M. A. Sanchez-Garcia, D. Basak, F. J. Sanchez, F. Calle, P. Youinou, E. Munoz, J. J. Serrano, J. M. Blanco, C. Villar, T. Laine, J. Oila, K. Saarinen, P. Hautojärvi, C. H. Molloy, D. J. Somerford, and I. Harrison, Phys. Rev. B 58, 1550 (1998).

${ }^{13}$ K. Saarinen, P. Seppälä, J. Oila, P. Hautojärvi, C. Corbel, O. Briot, and R. L. Aulombard, Appl. Phys. Lett. 73, 3253 (1998).

${ }^{14}$ T. Mattila and R. M. Nieminen, Phys. Rev. B 55, 9571 (1997).

${ }^{15}$ J. Neugebauer and C. Van de Walle, Appl. Phys. Lett. 69, 503 (1996).

${ }^{16}$ K. Saarinen, T. Suski, I. Grzegory, and D. C. Look, Phys. Rev. B 64, 233201 (2001).

${ }^{17}$ D. C. Look and J. R. Sizelove, Phys. Rev. Lett. 82, 1237 (1999).

${ }^{18}$ A. Uedono, S. F. Chichibu, Z. Q. Chen, M. Sumiya, R. Suzuki, T. Ohdaira, T. Mikado, T. Mukai, and S. Nakamura, J. Appl. Phys. 90, 181 (2001). 